\title{
Measuring barriers to adherence: validation of the problematic experiences of therapy scale
}

\author{
Sarah Kirby ${ }^{1}$, Maggie Donovan-Hall ${ }^{2}$, and Lucy Yardley ${ }^{1}$ \\ ${ }^{1}$ Academic Unit of Psychology, University of Southampton, Southampton, UK and ${ }^{2}$ Faculty of Health Sciences, University of Southampton, \\ Southampton, UK
}

\begin{abstract}
Purpose: To present the psychometric properties of the Problematic Experiences of Therapy Scale (the PETS), a brief measure to assess self-reported perceived barriers to adherence to physical rehabilitative therapy. Methods: Participants (study 1: $n=128$, study $2: n=227$ ) taking part in trials of rehabilitative exercises completed the PETS and adherence questions at 12 weeks. Participants in study 2 were also asked about maintained adherence at 6-month follow-up. Results: Principal component analysis identified a four-factor structure relating to symptoms, uncertainty, doubts and practical problems. Cronbach's alphas ranged between 0.84 and 0.96 for study 1 and study 2 . Correlations between factors varied, ranging between -0.22 and -0.53 for study 1 , and 0.12 and 0.36 for study 2 . Adherence was associated with all subscales at 12 weeks, and with the symptoms and doubts subscales at 6 -months. Conclusions: The PETS is a valid and reliable measure that can be used to assess participants' perceived reasons for non-adherence to a home-based rehabilitative therapy. It can be easily incorporated into treatment trials and as subscales were associated with reported adherence and maintained adherence, it provides potentially valuable indicators of reported barriers to adherence or might be used in clinical practice to facilitate conversations about adherence.
\end{abstract}

\section{> Implications for Rehabilitation}

- Low levels of adherence are commonly reported among people with chronic conditions who are required to undertake self-managed, home-based rehabilitation, yet patientperceived barriers to adherence are rarely measured.

- The Problematic Experiences of Therapy Scale (the PETS) is a brief self-report measure that assesses the extent to which respondents perceive that they have been prevented from carrying out an intervention by common and plausible reasons.

- A patient-centered approach to reasons for non-adherence could facilitate conversations about adherence and identify areas in which the respondent may benefit from additional support or interventions to aid adherence.

\author{
Keywords \\ Compliance, exercise, problematic \\ experiences of therapy scale, reliability, \\ validity
}

History

Received 29 May 2013

Revised 9 December 2013

Accepted 12 December 2013

Published online 13 January 2014

\section{Introduction}

Adherence to treatment is a complex issue that has generated a great deal of attention and has been highlighted as one of the most serious problems facing medical practice [1,2]. The consequences of non-adherence are considerable; it can affect the effectiveness and outcome of treatment, increase the amount of time spent with health care professionals and increase the financial cost of health care [3].

Literature reviews show that the highest levels of nonadherence are most often reported for patients with chronic conditions and patients undergoing treatments that require maintenance of behavioral and lifestyle changes [4-6].

Address for correspondence: Dr Sarah Kirby, Academic Unit of Psychology, University of Southampton, Highfield, Southampton, SO17 1BJ, UK. E-mail: sek@soton.ac.uk
Although non-adherence rates vary due to methodological and intervention factors [7], in home-based interventions where patients are required to self-manage their rehabilitation program, non-adherence rates have been reported to be as high as $63 \%-70 \%$ [8-10]. It is therefore important to identify the specific barriers to adherence reported in the context of different interventions, so that future interventions and treatment programs can be created or adapted to address these aspects [11-15].

One approach that has been advocated in attempting to improve self-management is a bottom-up, patient-centered approach [16,17]. The patient-centered approach encourages clinicians to be guided by the patients' knowledge and experience, and address patient concerns in a continuing dialog $[16,18,19]$. Quality of life can be defined in many different ways, and patients may judge their quality of life using different definitions from clinicians. Treatment side-effects and other consequences of treatment may impinge on factors which patients value as quality of life [20,21]. As such, patients' appraisal of their treatment 
experience may not be rational [22] and so may not necessarily be obvious to clinicians. Therefore, by taking a patient-centered approach to adherence, patients' views and reasons for nonadherence offer a valuable perspective to be taken into account alongside other perspectives [17,23,24], such as those of the clinician and evidence-based factors identified within research and models of behavior change $[25,26]$.

Validated scales exist that are based on a patient-centered, quantitative self-report approach to measure barriers to adherence to medication $[27,28]$; however, no validated measures exist to assess self-reported reasons for non-adherence to rehabilitation in a home-based setting. Measures do exist that assess the perspective of the health provider in relation to adherence [29], participation [30] and engagement [31] with rehabilitation in a treatment-based setting. Few studies explicitly ask patients to report what they felt were the specific reasons for their non-adherence, and studies that do this typically employ qualitative methods [32-34]. One such study that employed quantitative methods to do this was carried out by Leijon and colleagues [14], who followed up patients who did not adhere to their physical activity prescription. They asked participants to select one reason for their non-adherence from a list comprising sickness, pain, low motivation, lack of time, economic factors or another reason (but patients were not required to specify what this reason was). They found that low motivation was the most common reason for non-adherence to home-based activities, with older people being more likely to report sickness and pain, and younger people being more likely to report economic factors and lack of time. Whilst this study did explicitly ask the patients their perceived reasons for non-adherence, it is limited by its restriction to only one reason for non-adherence (those who gave more than one answer were excluded for providing an "invalid"' answer), and also did not allow for a scaled response.

The decision not to adhere is most likely to be multi-factorial [5]. Patients may encounter a variety of barriers and weigh up the overall costs and benefits of continuing with therapy $[35,36]$. There is also some evidence to suggest that non-adherence can be caused by the presence of one particular barrier during therapy that they were not able or willing to endure or overcome [37]. Measures of barriers to adherence are needed that take account of both possibilities.

The Problematic Experiences of Therapy Scale (PETS) is a brief quantitative measure that was developed based on clinical experience and research to reflect the most commonly reported reasons for discontinuing therapy given by patients undergoing self-managed home-based rehabilitation [9,38-40]. Although the PETS has been reported to be associated with adherence [10], the psychometric properties of the measure have not yet been published. Therefore, the aims of this paper were to use exploratory factor analysis to examine the structural and internal validity of the PETS, and examine whether the PETS was related to adherence and maintained the adherence.

\section{Methods}

\section{Participants and procedures}

Data were collected within the context of two randomized controlled trials of rehabilitation exercises for dizziness. The full details of the intervention and methods are reported with these trials (study 1: [41]; study 2: [10]).

Participants in study $1(n=128)$ were instructed to carry out rehabilitation exercises for 12 weeks or until their symptoms ceased (if this was sooner), and completed questionnaires pre- and post-treatment. Participants completed the PETS as part of their 12-week post-treatment assessment, followed by questions about adherence. Ethical approval was given by the South East and South West Local Research Ethics Committees, and written informed consent was given by all participants.

Data in study 2 were collected from 227 participants who were instructed to carry out exercises for 12 weeks or until their symptoms ceased. Questionnaires were completed at baseline, post-treatment, and 6-month follow-up. Participants completed the PETS as part of their 12-week post-treatment assessment, followed by questions about adherence. A question about maintained adherence was included in the 6-month follow-up. Ethical approval was obtained from the Ethical Committee at the School of Psychology, University of Southampton, and written informed consent was given by all participants.

\section{Measures}

\section{The PETS}

The PETS (see the Appendix) asks respondents the extent to which they agree that they have been prevented from carrying out an intervention by plausible reasons. Questions are worded this way in order to directly assess perceived reasons for non-adherence, rather than correlates of non-adherence, i.e. instead of asking respondents if the therapy made their symptoms worse, the PETS asks if the therapy making their symptoms worse was a reason why they did not carry out the therapy. It was developed to be used immediately prior to questions on adherence levels in randomized controlled trials of home-based rehabilitation exercises for dizziness [10,41,42]. The scale comprises 12 items divided into four subscales: "symptoms too severe or aggravated by therapy", (items 1-3), "uncertainty about how to carry out the treatment" (items 4-5), "doubts about treatment efficacy" (items 6-8), and "practical problems" such as lack of time or opportunity, forgetting (items 9-12). All items are scored on a scale ranging from 1 (disagree strongly) to 5 (agree strongly).

The subscales can be calculated in two ways. The first is to add the relevant items together and divide by the number of items in that subscale. This method gives an indication of the cumulative quantity and magnitude of perceived barriers encountered during therapy. The second method is to recode the scores into binary categories. Participants who respond "strongly disagree" to all items in a subscale are recoded as "no barriers", and all other scores are recoded as "some barriers or doubts". This method reflects the assumption that only one reason, no matter how strongly felt, may be a sufficient threshold to reduce or prevent adherence. The analyses reported in this paper use the binary method. For an example of the cumulative scoring method, see the results reported in Yardley and Kirby [10].

\section{Adherence}

This study classifies adherence in the same way as was reported in study 1 [41] and study 2 [10]. Participants were classified as demonstrating high adherence if they reported that they had carried out the exercises for at least 9 weeks or until they were no longer experiencing symptoms (if less than 9 weeks). Participants who reported that they had carried out the exercises for less than 9 weeks and were still experiencing symptoms were classified as low adherers.

\section{Maintained adherence}

In study 2, at 6-month follow up participants were asked if they had continued with the exercises after the 12-week period. Participants who answered yes to this question (irrespective of duration) were considered to have continued with the therapy. 


\section{Data analysis}

Data were analyzed using IBM SPSS Statistics for Windows (version 19.0, IBM Corp., Armonk, NY). Principal component analysis was used to assess the factor structure of the PETS. The suitability of the data for principal component analysis was checked using the Kaiser-Meyer-Olkin test and Bartlett's test of sphericity. As we expected factors to be correlated, oblique (direct oblimin) rotation was used $($ delta $=0)$. The number of factors to be retained was determined by three criteria: the a priori hypothesis that there would be four subscales, the scree plot, and the interpretability of the factor solution. Hypothesized factors were retained if eigenvalues were over 0.7 [43,44]. Factors were interpreted by items with loadings greater than 0.55 [45]. Internal consistency of the PETS subscales was assessed using Cronbach's alpha.

The PETS subscales were recoded into binary categories (as described above in the measures section; due to non-normal distributions). For each of the PETS subscales, differences between adherers and non-adherers (study 1), and those who did and did not continue with the treatment (study 2) were calculated using Chi-square tests.

\section{Results}

\section{Factor structure}

Principal component analysis for study 1 showed that the data were best represented by a four-factor solution corresponding to the four hypothesized subscales. These four factors accounted for $84 \%$ of the variance. Table 1 shows that all items clearly loaded onto one factor each, with loadings of 0.67 or more on one factor and less than 0.10 on other factors. All factors had eigenvalues greater than 0.9 . The component correlation matrix presented in
Table 2 showed that the correlations between subscales ranged between -0.22 and -0.53 . The correlation between the perceived barriers due to symptoms and practical problems subscales was the smallest, and the subscales concerned with perceived barriers due to uncertainty and practical problems were the most highly correlated.

The principal component analysis for study 2 (see Table 1) found that a clear four-factor solution emerged, accounting for $81 \%$ of the variance. All items loaded onto exactly the same factors reported in study 1 . All factors had eigenvalues greater than 1, and all items had factor loadings of 0.60 or more on one factor and less than 0.11 on other factors. The correlations between the PETS subscales for study 2 are also presented in Table 2 and show a similar pattern of inter-correlations to study 1 , although correlations were smaller in size ranging from 0.12 to 0.36 (the smallest correlation was between perceived barriers due to symptoms and uncertainty, and the largest was between perceived barriers due to uncertainty and doubts).

\section{Internal consistency}

All the PETS subscales in both study 1 and study 2 demonstrated good internal consistency. The Cronbach's alphas are presented in Table 1 and ranged from 0.84 to 0.96 . Item analyses showed that all items were more highly correlated with their own subscale than other subscales.

\section{Relation of the PETS to adherence and maintained adherence}

In study 1, participants who reported some barriers or doubts during the treatment period relating to all PETS subscales also reported lower levels of adherence (i.e. participants who reported

Table 1. Principal component analysis (pattern matrix) and internal consistency of the PETS items.

\begin{tabular}{|c|c|c|c|c|c|c|c|c|}
\hline & \multicolumn{4}{|c|}{ Study $1(N=128)$} & \multicolumn{4}{|c|}{ Study $2(N=225)$} \\
\hline \multicolumn{9}{|l|}{ Symptoms too severe or aggravated by therapy ( $\alpha$ study 1 and study $2=0.91$ ) } \\
\hline I was prevented from carrying out the therapy by severe symptoms & -0.10 & -0.92 & -0.02 & -0.08 & 0.00 & 0.92 & 0.04 & 0.04 \\
\hline I could not carry out the therapy because it caused more symptoms & 0.05 & -0.92 & 0.01 & 0.00 & -0.03 & 0.91 & -0.05 & -0.04 \\
\hline \multicolumn{9}{|l|}{ Uncertainty about how to carry out the treatment $(\alpha$ study $1=0.96 ; \alpha$ study $2=0.93$ ) } \\
\hline \multicolumn{9}{|l|}{ Doubts about treatment efficacy ( $\alpha$ study $1=0.94 ; \alpha$ study $2=0.84$ ) } \\
\hline I skipped the therapy because I was not sure if it was helping & 0.92 & -0.07 & 0.04 & 0.04 & 0.07 & 0.10 & -0.79 & -0.06 \\
\hline I skipped the therapy because it did not seem relevant to my symptoms an & 0.91 & 0.06 & 0.02 & -0.09 & 0.02 & -0.09 & -0.89 & 0.02 \\
\hline I did not carry out the therapy because I was not convinced it was right for me & 0.95 & 0.02 & -0.04 & -0.02 & -0.07 & 0.04 & -0.90 & 0.04 \\
\hline \multicolumn{9}{|l|}{ Practical problems $(\alpha$ study $1=0.84 ; \alpha$ study $2=0.87)$} \\
\hline Lack of time prevented me from carrying out the therapy & -0.02 & -0.09 & 0.89 & 0.02 & 0.93 & -0.02 & 0.05 & 0.04 \\
\hline It was not possible to find suitable opportunities to carry out the therapy & 0.05 & 0.02 & 0.93 & 0.08 & 0.94 & -0.03 & 0.05 & .001 \\
\hline
\end{tabular}

Items included in each factor are shown in bold.

Table 2. Component correlation matrices of PETS subscales (study 1 and 2).

\begin{tabular}{|c|c|c|c|c|c|c|}
\hline & \multicolumn{3}{|c|}{ Study 1} & \multicolumn{3}{|c|}{ Study 2} \\
\hline & 1 & 2 & 3 & 1 & 2 & 3 \\
\hline 1. Symptoms too severe or aggravated by therapy & - & & & - & & \\
\hline 2. Uncertainty about how to carry out the treatment & 0.37 & - & & 0.12 & - & \\
\hline 3. Doubts about treatment efficacy & -0.40 & -0.50 & - & -0.18 & 0.36 & - \\
\hline 4. Practical problems & -0.22 & -0.53 & 0.34 & -0.13 & -0.32 & -0.25 \\
\hline
\end{tabular}


more barriers or doubts due to the PETS subscales were also more likely to report carrying out the exercises for less than 9 weeks and were still experiencing symptoms). For perceived barriers or doubts due to increased or aggravated symptoms, $39(47 \%)$ of those who reported some barriers or doubts were low adherers, compared with 7 (14.6\%) of those who strongly disagreed $\left(\chi^{2}=14.02, d f=1, n=131, p<0.001\right)$. For the uncertainty subscale, $25(51 \%)$ of those who reported some barriers or doubts were low adherers, compared with 23 (26.4\%) of those who strongly disagreed $\left(\chi^{2}=8.30, d f=1, n=136, p<0.01\right)$. For the doubts subscale, differences between the number of participants who were low adherers to treatment and reported some barriers or doubts, or strong disagreement was $32(50 \%)$ and $14(20 \%)$, respectively $\left(\chi^{2}=13.35, \quad d f=1, \quad n=134\right.$, $p<0.001)$. Finally, for perceived barriers or doubts due to practical problems, 36 (42.9\%) of those who reported some barriers or doubts were low adherers to treatment, compared with $13(25 \%)$ of those who strongly disagreed $\left(\chi^{2}=4.44, d f=1\right.$, $n=136, p<0.05)$.

In study 2, Yardley and Kirby [10] reported that all the PETS subscales could significantly differentiate between high and low adherers at the 12-week follow-up. In addition to this, participants who voluntarily continued with the treatment into the 6-month follow-up period $(n=106,47.5 \%)$ did not report any perceived barriers or doubts due to symptoms or doubts about treatment efficacy at 12 weeks (symptoms $\chi^{2}=8.62, d f=1, n=221$, $p<0.01$; doubts $\left.\chi^{2}=11.24, d f=1, n=222, p<0.01\right)$, suggesting that these factors may be more relevant to the maintenance of treatment.

\section{Discussion}

A range of validated and widely used self-report scales exist for adherence to medication [36,46,47], and clinic-based rehabilitation [29]; however, no self-report adherence measures exist for use in home-based rehabilitation. This paper describes the factor structure and internal consistency of the PETS, a brief quantitative measure designed to assess perceived barriers to adherence by asking respondents the extent to which they agree that they had been prevented from carrying out an intervention by plausible reasons. Structural analysis confirmed the four hypothesized subscales which all demonstrated either excellent or good internal consistency. Inter-correlations between subscales were modest, suggesting that whilst subscales were related, they each represented different types of perceived barriers.

The PETS was able to distinguish between high and low adherers in both study 1 (using the binary scoring method, reported in this paper) and study 2 (using the cumulative scoring method; reported in Yardley and Kirby [10], not reported in this paper). The PETS may also contribute to understanding factors relating to the maintenance of treatment adherence, as those who had voluntarily continued with their therapy at the 6-month follow-up in study 2 had reported less symptoms or doubts about treatment efficacy at 12 weeks.

Previous studies that elicit participants' perceived reasons for non-adherence have typically employed qualitative methods [32-34]. Although these in-depth methods are important for the understanding of the patient perspective, their findings cannot be easily integrated into statistical analyses of outcomes, and are more difficult to use in clinical practice [38]. The PETS could be used in clinical practice as a tool to facilitate conversations about adherence and identify areas in which the respondent may benefit from additional support or interventions to aid adherence. By assuming that patients will encounter difficulties in relation to specific rather than general issues, and by presenting such questions before asking patients about their actual adherence levels, a foundation is provided upon which a more open and honest disclosure and discussion about adherence can hopefully be based.

\section{Limitations and future research}

It is important to note that this paper and the PETS have several limitations. The PETS was initially developed based on clinical experience to be used in research trials immediately prior to selfreported adherence questions with the hope that it would reduce socially desirable responding. Therefore, although the item development was influenced by early literature and research [9,38-40], the items were not developed from a systematic review of literature or specific patient-based research carried out for that purpose. Therefore, the PETS may not capture all the factors relevant to perceived reasons for non-adherence. Further work is needed to identify and incorporate these factors using systematic methods. In addition, the hypothesis that use of the PETS before actual adherence questions would reduce socially desirable responding has not yet been tested and responses to the PETS may still be influenced by socially desirable responding. For example, participants may be reluctant to indicate that they did not understand what to do as they may feel that this implies that their therapist has not given satisfactory explanations. Another limitation of this paper with regards to the measurement of adherence, is that it is observational, since participants could only be allocated to groups (adherence versus non-adherence) based on their patterns of behavior - random allocation to these groups was not possible. It is also important to note that non-adherence is likely to be multi-factorial, many other factors have been reported to influence adherence and so may have been relevant to participants' patterns of behavior [4,5,15,48-50]. Therefore, whilst this paper assesses the independent relationship between the PETS and adherence, we can make no claim that these effects will remain when all other potentially relevant factors are statistically adjusted for, and future studies are needed to control for these factors. It was also beyond the scope of this paper to assess the test-retest reliability of the PETS. It would be useful to measure responses to the PETS at repeated points during a therapy to see if perceived reasons for non-adherence are consistent or vary across time or different stages of therapy. Future research should also explore the relationship of the PETS to objective measures of adherence and other adherence outcomes, as well as other illness populations and intervention types in clinical practice.

Despite these limitations, the PETS represents a reliable and valid measure that can be used to assess participants' given reasons for non-adherence to home-based rehabilitative therapy. The relationship between perceived barriers to adherence and adherence itself is a complex one [28]; however, our results suggest that the PETS may contribute some explanation as to the mechanism by which non-adherence occurs within the framework of a patient-centered approach.

\section{Declaration of interest}

The authors report no conflicts of interest. The authors alone are responsible for the content and writing of this article.

Study 1 was funded by grant SEO 083 from the Directorate of Health and Social Care South, London, United Kingdom. Study 2 was funded by a project grant from the Ménière's Society (UK).

\section{References}

1. Becker MH. Patient adherence to prescribed therapies. Medical Care 1985;23:539-55.

2. Clough BA, Casey LM. Technological adjuncts to increase adherence to therapy: a review. Clin Psychol Rev 2011;31:697-710. 
3. Myers LB, Midence K. Concepts and issues in adherence. In: Myers LB, Midence K, eds. Adherence to treatment in medical conditions. India: Harwood Academic Publishers; 1998:1-24.

4. DiMatteo MR. Variations in patients' adherence to medical recommendations: a quantitative review of 50 years of research. Med Care 2004:42:200-9.

5. Sabate E. Adherence to long-term therapies: evidence for action. Geneva, Switzerland: World Health Organisation; 2003.

6. van Dulmen S, Sluijs E, van Dijk L, et al. Patient adherence to medical treatment: a review of reviews. BMC Health Serv Res 2007; 7:55. doi:10.1186/1472-6963-7-55.

7. Fjeldsoe B, Neuhaus M, Winkler E, Eakin E. Systematic review of maintenance of behavior change following physical activity and dietary interventions. Health Psychol 2011;30:99-109.

8. Shang J, Wenzel J, Krumm S, et al. Who will drop out and who will drop in exercise adherence in a randomized clinical trial among patients receiving active cancer treatment. Cancer Nurs 2012;35: 312-22.

9. Sluijs EM, Kok GJ, van der Zee J. Correlates of exercise compliance in physical therapy. Phys Ther 1993;73:771-86.

10. Yardley L, Kirby S. Evaluation of booklet-based self-management of symptoms in Ménière disease: a randomized controlled trial. Psychosom Med 2006;68:762-9.

11. DiMatteo M, Haskard-Zolnierek KB, Martin LR. Improving patient adherence: a three-factor model to guide practice. Health Psychol Rev 2012;6:74-91.

12. Martin KA, Sinden AR. Who will stay and who will go? A review of older adults' adherence to randomized controlled trials of exercise. J Aging Phys Activity 2001;9:91-114.

13. Lequerica $\mathrm{AH}$, Kortte $\mathrm{K}$. Therapeutic engagement a proposed model of engagement in medical rehabilitation. Am J Phys Med Rehabil 2010;89:415-22.

14. Leijon ME, Faskunger J, Bendtsen P, et al. Who is not adhering to physical activity referrals, and why? Scand J Primary Health Care 2013;29:234-40.

15. Jack K, Mclean SM, Moffett JK, Gardiner E. Barriers to treatment adherence in physiotherapy outpatient clinics: a systematic review. Manual Therapy 2010;15:220-8.

16. Martin LR, Haskard-Zolnierek KB, DiMatteo MR. Health behaviour change and treatment adherence. Oxford: Oxford University Press; 2010.

17. Rogers A, Kennedy A, Nelson E, Robinson A. Uncovering the limits of patient-centeredness: implementing a selfmanagement trial for chronic illness. Qualitative Health Res 2005; 15:224-39.

18. Byrne PS, Long BEL. Doctors talking to patients. London: HMSO; 1976.

19. Stiggelbout A, Van der Weijden T, De Wit M, et al. Shared decision making: really putting patients at the centre of healthcare. Brit Medical J 2012;344:e256.

20. Crossley ML. Rethinking health psychology. Oxford: Open University Press; 2000.

21. Turk DC, Rudy TE. Neglected topics in the treatment of chronic pain patients - relapse, noncompliance and adherence enhancement. Pain 1991;44:5-28.

22. Redelmeier DA, Rozin P, Kahneman D. Understanding patients decisions - cognitive and emotional perspectives. J Am Medical Assoc 1993;270:72-6.

23. Mead N, Bower P. Patient-centredness: a conceptual framework and review of the empirical literature. Sol Sci Medicine 2000;51: 1087-110.

24. Cott CA. Client-centred rehabilitation: client perspectives. Disabil Rehabil 2004;26:1411-22.

25. Abraham C, Michie S. A taxonomy of behavior change techniques used in interventions. Health Psychol 2008;27:379-87.

26. Michie S, Johnston M, Francis J, et al. From theory to intervention: mapping theoretically derived behavioural determinants to behaviour change techniques. Appl Psychol - An Int Rev-Psycholog Appliquee-Revue Internationale 2008;57:660-80.
27. Svarstad BL, Chewning BA, Sleath BL, Claesson C. The brief medication questionnaire: a tool for screening patient adherence and barriers to adherence. Patient Educ Counsel 1999;37:113-24.

28. Wetzels G, Nelemans P, van Wijk B, et al. Determinants of poor adherence in hypertensive patients: development and validation of the "Maastricht Utrecht Adherence in Hypertension (MUAH)questionnaire', Patient Educ Counseling 2006;64:151-8.

29. Brewer BW, Van Raalte JL, Petitpas AJ, et al. Preliminary psychometric evaluation of a measure of adherence to clinic-based sport injury rehabilitation. Phys Therapy in Sport 2000;1:68-74.

30. Lenze EJ, Munin MC, Quear T, et al. The Pittsburgh rehabilitation participation scale: reliability and validity of a clinician-rated measure of participation in acute rehabilitation. Arch Phys Medicine Rehabil 2004;85:380-4.

31. Kortte KB, Falk LD, Castillo RC, et al. The Hopkins rehabilitation engagement rating scale: development and psychometric properties. Arch Phys Med Rehabil 2007;88:877-84.

32. Courneya KS, McKenzie DC, Reid RD, et al. Barriers to supervised exercise training in a randomized controlled trial of breast cancer patients receiving chemotherapy. Ann Behav Med 2008;35:116-22.

33. Jancey JM, Clarke A, Howat P, et al. Perceptions of physical activity by older adults: a qualitative study. Health Educ J 2009;68:196-206.

34. Tierney S, Mamas M, Skelton D, et al. What can we learn from patients with heart failure about exercise adherence? A systematic review of qualitative papers. Health Psychol 2011;30:401-10.

35. Wroe AL. Intentional and unintentional nonadherence: a study of decision making. J Behav Med 2002;25:355-72.

36. Horne R, Weinman J, Hankins M. The beliefs about medicines questionnaire: the development and evaluation of a new method for assessing the cognitive representation of medication. Psychol Health 1999; 14:1-24.

37. Jones M, Jolly K, Raftery J, et al., BRUM Steering Committee. 'DNA' may not mean 'did not participate': a qualitative study of reasons for non-adherence at home- and centre-based cardiac rehabilitation. Family Pract 2007;24:343-57.

38. Campbell R, Evans M, Tucker M, et al. Why don't patients do their exercises? Understanding non-compliance with physiotherapy in patients with osteoarthritis of the knee. J Epidemiol Commun Health 2001;55:132-8.

39. Yardley L, Beech S, Zander L, et al. A randomized controlled trial of exercise therapy for dizziness and vetigo in primary care. Brit J General Pract 1998;48:1136-40.

40. Yardley L, Sharples K, Beech S, Lewith G. Developing a dynamic model of treatment perceptions. J Health Psychol 2001;6:269-82.

41. Yardley L, Donovan-Hall M, Smith HE, et al. Effectiveness of primary care-based vestibular rehabilitation for chronic dizziness. Annals Internal Med 2004;141:598-605.

42. Yardley L, Barker F, Muller I, et al. Clinical and cost effectiveness of booklet based vestibular rehabilitation for chronic dizziness in primary care: single blind, parallel group, pragmatic, randomised controlled trial. Brit Med J 2012;344:e2237.

43. Jolliffe IT. Discarding variables in a principal component analysis. I: artificial data. J Royal Statist Soc Series C (Appl Statist 1972;21: $160-73$.

44. Jolliffe IT. Principal component analysis. 2nd ed. New York: Springer-Verlag; 2002.

45. Comrey AL, Lee HB. A first course in factor analysis. 2nd ed. Hillsdale (NJ): Lawrence Erlbaum Associates; 1992.

46. Morisky DE, Green LW, Levine DM. Concurrent and predictivevalidity of a self-reported measure of medication adherence. Medical Care 1986;24:67-74.

47. Thompson K, Kulkarni J, Sergejew AA. Reliability and validity of a new Medication Adherence Rating Scale (MARS) for the psychoses Schizophrenia Res 2000;42:241-7.

48. Conner M, Norman P. Predicting health behaviour. 2nd ed Buckingham, UK: Open University Press; 2005.

49. DiMatteo MR. Social support and patient adherence to medical treatment: a meta-analysis. Health Psychol 2004;23:207-18.

50. Meichenbaum D, Turk DC. Facilitating treatment adherence: a practitioner's guidebook. New York: Plenum Press; 1987. 


\section{Appendix. The Problematic Experiences of Therapy Scale (PETS)}

We would like to know how easy or difficult it was for you to carry out the therapy. We want to find out if it was difficult in any way for you to carry out at home, and if so, what difficulties were and how often they prevented you from practicing the techniques you have learnt. Please circle the most appropriate response.

Problems due to symptoms

1 I had to skip [the therapy]* because it made my symptoms worse.

2 I was prevented from [carrying out the therapy] by severe symptoms.

3 I could not [carry out the therapy] because it caused more symptoms.

Problems due to uncertainty or doubts about the therapy

4 I could not [carry out the therapy] because I was unsure how to do it properly.

5 I was unable to [carry out the therapy] because it was difficult to know what to do.

6 I skipped [the therapy] because I was not sure if it was helping.

7 I skipped [the therapy] because it did not seem relevant to my symptoms and problems.

8 I did not [carry out the therapy] because I was not convinced it was right for me.

Practical problems

9 Lack of time prevented me from [carrying out the therapy].

10 It was not possible to find suitable opportunities to [carry out the therapy].

11 I was too busy or tired to [carry out the therapy].

12 I found it difficult to remember to [carry out the therapy].

\begin{tabular}{|c|c|c|c|c|}
\hline Agree Strongly & Agree Slightly & Not Sure & Disagree Slightly & Disagree Strongly \\
\hline Agree Strongly & Agree Slightly & Not Sure & Disagree Slightly & Disagree Strongly \\
\hline Agree Strongly & Agree Slightly & Not Sure & Disagree Slightly & Disagree Strongly \\
\hline Agree Strongly & Agree Slightly & Not Sure & Disagree Slightly & Disagree Strongly \\
\hline Agree Strongly & Agree Slightly & Not Sure & Disagree Slightly & Disagree Strongly \\
\hline Agree Strongly & Agree Slightly & Not Sure & Disagree Slightly & Disagree Strongly \\
\hline Agree Strongly & Agree Slightly & Not Sure & Disagree Slightly & Disagree Strongly \\
\hline Agree Strongly & Agree Slightly & Not Sure & Disagree Slightly & Disagree Strongly \\
\hline Agree Strongly & Agree Slightly & Not Sure & Disagree Slightly & Disagree Strongly \\
\hline Agree Strongly & Agree Slightly & Not Sure & Disagree Slightly & Disagree Strongly \\
\hline $\begin{array}{l}\text { Agree Strongly } \\
\text { A gree Strongly }\end{array}$ & Agree Slightly & Not Sure & Disagree Slightly & Disagree Strongly \\
\hline Agree Strongly & Agree Slightly & Not Sure & Disagree Slightly & Disagree Strongly \\
\hline
\end{tabular}

*References to "the therapy" can be modified to fit the situation the PETS is used in. 